\title{
Detection of supernova neutrinos by neutrino-proton elastic scattering
}

\author{
John F. Beacom* \\ NASA/Fermilab Astrophysics Center, Fermi National Accelerator Laboratory, Batavia, Illinois 60510-0500
}

Will M. Farr ${ }^{\dagger}$ and Petr Vogel ${ }^{\ddagger}$

Physics Department 161-33, Caltech, Pasadena, California 91125

(Received 20 May 2002; published 1 August 2002)

\begin{abstract}
We propose that neutrino-proton elastic scattering, $\nu+p \rightarrow \nu+p$, can be used for the detection of supernova neutrinos in scintillator detectors. Though the proton recoil kinetic energy spectrum is soft, with $T_{p}$ $\simeq 2 E_{v}^{2} / M_{p}$, and the scintillation light output from slow, heavily ionizing protons is quenched, the yield above a realistic threshold is nearly as large as that from $\bar{\nu}_{e}+p \rightarrow e^{+}+n$. In addition, the measured proton spectrum is related to the incident neutrino spectrum, which solves a long-standing problem of how to separately measure the total energy and temperature of $\nu_{\mu}, \nu_{\tau}, \bar{\nu}_{\mu}$, and $\bar{\nu}_{\tau}$. The ability to detect this signal would give detectors like KamLAND and Borexino a crucial and unique role in the quest to detect supernova neutrinos.

DOI: 10.1103/PhysRevD.66.033001

PACS number(s): 13.15.+g, 97.60.Bw
\end{abstract}

\section{INTRODUCTION}

When the next Galactic supernova occurs, approximately $10^{4}$ detected neutrino events are expected among the several detectors around the world. It is widely believed that these $10^{4}$ events will provide important clues to the astrophysics of the supernova as well as the properties of the neutrinos themselves. Interestingly, recent breakthroughs in understanding solar and atmospheric neutrinos each occurred when the accumulated samples of detected events first exceeded $10^{4}$.

But will we have enough information to study the supernova neutrino signal in detail? Almost all of the detected events will be charged current $\bar{\nu}_{e}+p \rightarrow e^{+}+n$, which will be well measured, both because of the large yield and because the measured positron spectrum is closely related to the neutrino spectrum. Because of the charged-lepton thresholds, the flavors $\nu_{\mu}, \nu_{\tau}, \bar{\nu}_{\mu}$, and $\bar{\nu}_{\tau}$ can only be detected in neutralcurrent reactions, of which the total yield is expected to be approximately $10^{3}$ events. However, as will be discussed below, in general one cannot measure the neutrino energy in neutral-current reactions. This paper presents an exception. These four flavors are expected to carry away about $2 / 3$ of the supernova binding energy, and are expected to have a higher temperature than $\nu_{e}$ or $\bar{\nu}_{e}$. However, there is no experimental basis for these statements, and at present, numerical models of supernovae cannot definitively address these issues either. If there is no spectral signature for the neutralcurrent detection reactions, then neither the total energy carried by these flavors nor their temperature can be separately determined from the detected number of events.

But it is crucial that these quantities be measured. Both are needed for comparison to numerical supernova models. The total energy is needed to determine the mass of the neutron star, and the temperature is needed for studies of neu-

\footnotetext{
*Email address: beacom@fnal.gov

${ }^{\dagger}$ Email address: farr@its.caltech.edu

‡Email address: vogel@citnp.caltech.edu
}

trino oscillations. At present, such studies would suffer from the need to make model-dependent assumptions. This problem has long been known, but perhaps not widely enough appreciated. In this paper, we clarify this problem, and provide a realistic solution that can be implemented in two detectors, KamLAND (already operating) and Borexino (to be operating soon). The solution is based on neutrino-proton elastic scattering, which has been observed at accelerators at $\mathrm{GeV}$ energies, but has never before been shown to be a realistic detection channel for low-energy neutrinos. Some of our preliminary results have been reported at conferences [1].

\section{CROSS SECTION}

The cross section for neutrino-proton elastic scattering is an important prediction [2] of the standard model, and it has been confirmed by extensive measurements at $\mathrm{GeV}$ energies (see, e.g., Ref. [3]). At the energies considered here, the full cross section formula [2-4] can be greatly simplified. At low energies, the differential cross section as a function of neutrino energy $E_{\nu}$ and struck proton recoil kinetic energy $T_{p}$ (and mass $M_{p}$ ) is

$$
\begin{aligned}
\frac{d \sigma}{d T_{p}}= & \frac{G_{F}^{2} M_{p}}{2 \pi E_{\nu}^{2}}\left[\left(c_{V}+c_{A}\right)^{2} E_{\nu}^{2}+\left(c_{V}-c_{A}\right)^{2}\left(E_{\nu}-T_{p}\right)^{2}\right. \\
& \left.-\left(c_{V}^{2}-c_{A}^{2}\right) M_{p} T_{p}\right] .
\end{aligned}
$$

The neutral-current coupling constants between the exchanged $Z^{\circ}$ and the proton are

$$
\begin{aligned}
& c_{V}=\frac{1-4 \sin ^{2} \theta_{w}}{2}=0.04, \\
& c_{A}=\frac{1.27}{2},
\end{aligned}
$$

where the factor 1.27 is determined by neutron beta decay and its difference from unity is a consequence of the partially 
conserved axial vector current. Equation (2.1) may be obtained directly by summing the contributions from the valence quarks. The cross section for antineutrinos is obtained by the substitution $c_{A} \rightarrow-c_{A}$. At high energies, the primitive couplings are functions of $q^{2} / M^{2}$, where $M \sim 1 \mathrm{GeV}$ (the proton mass or the dipole form-factor masses); since $q^{2}$ $=2 M_{p} T_{p} \sim E_{\nu}^{2}$, this variation may be safely neglected here. At order $E_{\nu} / M_{p}$, there is also a weak magnetism term which we have neglected. This would appear inside the square brackets in Eq. (2.1) as

$$
4 T_{p} E_{\nu} c_{M} c_{A}
$$

where $c_{M} \simeq 1.4$ depends on the proton and neutron magnetic moments [4]. This term is thus positive for neutrinos and negative for antineutrinos. In addition to being numerically small (less than a 10\% correction), this term will cancel in the measured differential cross section due to the indistinguishable contributions of neutrinos and antineutrinos. For $\nu_{\mu}$ and $\nu_{\tau}$, we assume the same fluxes and spectra for particles and antiparticles (as well as each other); the weak magnetism term above causes small corrections to the emitted spectra [5] that we can neglect here. For $\nu_{e}$ and $\bar{\nu}_{e}$, the expected fluxes and spectra are different from each other, but at the lower energies of these flavors the whole correction is very small. Other than the above points, Eq. (2.1) is correct to all orders in $E_{\nu} / M_{p}$. As will be emphasized below, our results are totally independent of oscillations among active flavors, as this is a neutral-current reaction.

We use the struck proton kinetic energy in the laboratory frame as our kinematic variable. For a neutrino energy $E_{\nu}$, $T_{p}$ ranges between 0 and $T_{p}^{\max }$, where

$$
T_{p}^{\max }=\frac{2 E_{v}^{2}}{M_{p}+2 E_{\nu}} \simeq \frac{2 E_{v}^{2}}{M_{p}} .
$$

The maximum is obtained when the neutrino recoils backwards with its original momentum $E_{\nu}$, and thus the proton goes forward with momentum $2 E_{\nu}$. The other kinematic variables can be related to $T_{p}$, and are

$$
\begin{aligned}
& \cos \theta_{p}=\frac{E_{\nu}+M_{p}}{E_{\nu}} \sqrt{\frac{T_{p}}{T_{p}+2 M_{p}}} \simeq \sqrt{\frac{M_{p} T_{p}}{2 E_{\nu}^{2}}} \\
& \cos \theta_{\nu}=1-\frac{M_{p} T_{p}}{E_{\nu}\left(E_{\nu}-T_{p}\right)} \simeq 1-\frac{M_{p} T_{p}}{E_{\nu}^{2}},
\end{aligned}
$$

where $\theta_{p}$ and $\theta_{\nu}$ are the angles of the final proton and neutrino with respect to the direction of the incident neutrino. In a scintillator-based detector, the proton direction cannot be measured, so these expressions are useful just for checking the cross section and kinematics.

If we take $\left(E_{\nu}-T_{p}\right)^{2} \simeq E_{\nu}^{2}$ (i.e., keeping only the lowest order in $E_{\nu} / M_{p}$, a very good approximation), then the differential cross section is very simple:

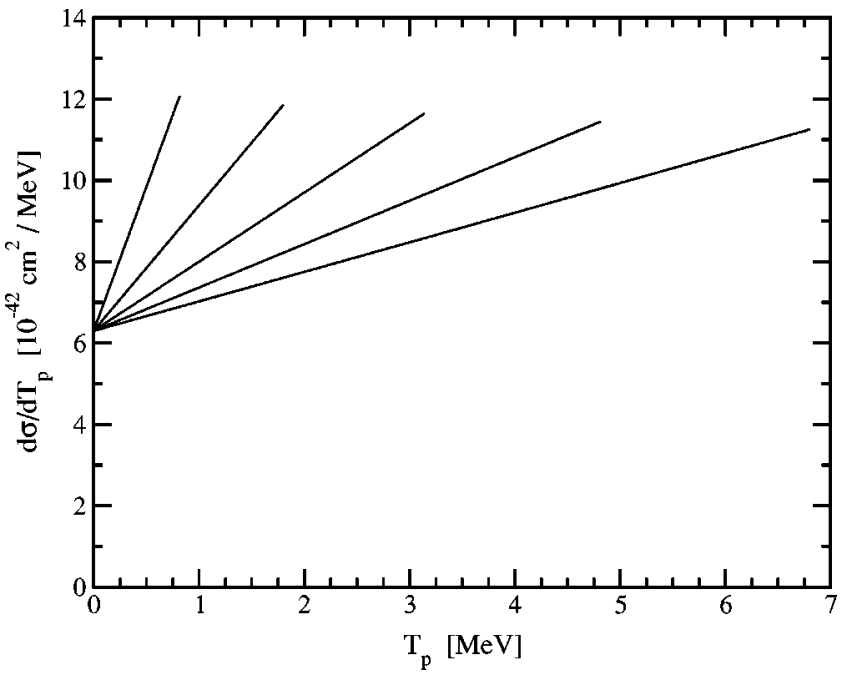

FIG. 1. The differential cross section as a function of $T_{p}$ for fixed $E_{\nu}$. Note the rise at large $T_{p}$, indicating that large kinetic energies are preferred. From left to right, the lines are for $E_{\nu}=20$, 30, 40, 50, and $60 \mathrm{MeV}$.

$$
\frac{d \sigma}{d T_{p}}=\frac{G_{F}^{2} M_{p}}{\pi}\left[\left(1-\frac{M_{p} T_{p}}{2 E_{\nu}^{2}}\right) c_{V}^{2}+\left(1+\frac{M_{p} T_{p}}{2 E_{\nu}^{2}}\right) c_{A}^{2}\right] .
$$

Since $c_{A} \gg c_{V}$, this form makes it clear that the largest proton recoils are favored, which is optimal for detection. Plots of $d \sigma / d T_{p}$ for fixed $E_{\nu}$ are shown in Fig. 1. Note that these slope in the opposite sense of the corresponding $d \sigma / d T_{e}$ curves for $\nu_{\mu}-e^{-}$scattering. The difference is simply due to the very different kinematics. For neutrino-proton elastic scattering, $T_{p}^{\max } \simeq 2 E_{\nu}^{2} / M_{p} \ll E_{\nu}$, while for neutrino-electron scattering, $T_{e}^{\max } \simeq E_{\nu}$. In this limit, the neutrino $\left(c_{A}\right)$ and antineutrino $\left(-c_{A}\right)$ cross sections are identical. If $c_{V}$ is neglected and the differential cross section is expressed in terms of $\cos \theta_{\nu}$, it follows the form $1-1 / 3 \cos \theta_{\nu}$ expected for a nonrelativistic axial vector coupling (i.e., a GamowTeller matrix element). The total cross section is

$$
\frac{G_{F}^{2} E_{v}^{2}}{\pi}\left(c_{V}^{2}+3 c_{A}^{2}\right)
$$

As expected, this is of the same form as the total cross section for the charged-current reaction $\bar{\nu}_{e}+p \rightarrow e^{+}+n$ (see, e.g., Ref. [6]). In the neutral-current case, the vector coupling nearly vanishes, and the axial vector coupling is half as large as in the charged-current channel, making the total cross section approximately 4 times smaller. This factor of 4 can be immediately obtained by considering the product of the couplings and the propagator factor, and using the definition of $\theta_{W}$.

It is also interesting to compare the neutrino-proton elastic scattering cross section with that for neutrino-electron elastic scattering (for $\nu_{\mu}$ so that only the neutral-current part is compared). Again, the different kinematics, reflected in the maximum kinetic energies, are crucial. The cross section for neutrino-electron scattering is much smaller: 


$$
\frac{\sigma_{t o t}\left(\nu_{\mu}+e^{-}\right)}{\sigma_{t o t}\left(\nu_{\mu}+p\right)} \sim \frac{G_{F}^{2} E_{\nu} m_{e}}{G_{F}^{2} E_{\nu}^{2}} \sim \frac{m_{e}}{E_{\nu}},
$$

which is $\sim 10^{-2}$ for our range of energies. ${ }^{1}$

In the above expressions, we have neglected contributions from strange sea quarks in the proton [7]. Strange-quark effects can enter Eq. (2.1) in three ways [8]. First, the vector form factor $c_{V}$ is modified by the strangeness charge radius squared $\left\langle r_{s}\right\rangle^{2}$ by a term proportional to $q^{2}\left\langle r_{s}\right\rangle^{2}$. Since our $q^{2}$ is so low, this is negligible. Second, the magnetic form factor $c_{M}$ is modified by the strange magnetic moment of the proton $\mu_{s}$. This is numerically small, and appears only in the small weak magnetism correction (see above). Third, the strange-quark contribution $\Delta s$ to the nucleon spin gives an isoscalar contribution to the axial vector form factor $c_{A}$, as

$$
c_{A} \rightarrow c_{A}=\frac{1.27}{2}-\frac{\Delta s}{2} .
$$

The rule for the cross section given above, of using $c_{A}$ for neutrinos and $-c_{A}$ for antineutrinos, is also true for the combined $c_{A}$ expression given here [8]. The value of $\Delta s$ is very poorly known from experiment, and is perhaps $\Delta s=-0.15$ \pm 0.15 [8]. Since $c_{A} \gg c_{V}$, this could increase the differential cross section by approximately $30 \%$, with an uncertainty of the same size. It is important to note that the $\Delta s$ contribution would not change the shape of the differential cross section, since $c_{A} \gg c_{V}$. It may be possible to measure $\Delta s$ directly via neutrino-proton elastic scattering at $\sim 1 \mathrm{GeV}$ in MiniBooNE [9].

\section{SUPERNOVA NEUTRINOS}

In this paper, we characterize the supernova neutrino signal in a very simple way, though consistently with numerical supernova models [10]. The change in gravitational binding energy between the initial stellar core and the final protoneutron star is about $3 \times 10^{53}$ ergs, about $99 \%$ of which is carried off by all flavors of neutrinos and antineutrinos over about $10 \mathrm{~s}$. The emission time is much longer than the lightcrossing time of the proto-neutron star because the neutrinos are trapped and must diffuse out, eventually escaping with approximately Fermi-Dirac spectra characteristic of the surface of last scattering. In the usual model, $\nu_{\mu}, \nu_{\tau}$ and their antiparticles are emitted with temperature $T \simeq 8 \mathrm{MeV}, \bar{\nu}_{e}$ has $T \simeq 5 \mathrm{MeV}$, and $\nu_{e}$ has $T \simeq 3.5 \mathrm{MeV}$. The temperatures differ from each other because $\bar{\nu}_{e}$ and $\nu_{e}$ have chargedcurrent opacities (in addition to the neutral-current opacities common to all flavors), and because the proto-neutron star has more neutrons than protons. It is generally assumed that

\footnotetext{
${ }^{1}$ It is interesting to note that the total rates of neutrino-proton elastic scattering events from solar neutrinos are huge: in the $1 \mathrm{kton}$ KamLAND detector, the rates from the $p p,{ }^{7} \mathrm{Be}$, and ${ }^{8} \mathrm{~B}$ fluxes are very roughly $10^{3} /$ day, $10^{3} /$ day, and $10^{2} /$ day, respectively; however, these are only at very low (unquenched) proton kinetic energies of approximately $0.2 \mathrm{keV}, 2 \mathrm{keV}$, and $200 \mathrm{keV}$, respectively.
}

each of the six types of neutrino and antineutrino carries away about $1 / 6$ of the total binding energy, though this has an uncertainty of at least $50 \%$ [11]. The supernova rate in our Galaxy is estimated to be $(3 \pm 1)$ per century (this is reviewed in Ref. [12]).

The expected number of events (assuming a hydrogen to carbon ratio in the detector of $2: 1$ ) is

$$
\begin{aligned}
N= & 70.8\left[\frac{E}{10^{53} \mathrm{erg}}\right]\left[\frac{1 \mathrm{MeV}}{T}\right]\left[\frac{10 \mathrm{kpc}}{D}\right]^{2}\left[\frac{M_{D}}{1 \mathrm{kton}}\right] \\
& \times\left[\frac{\langle\sigma\rangle}{10^{-42} \mathrm{~cm}^{2}}\right] .
\end{aligned}
$$

(Though written slightly differently, this is equivalent to the similar expression in Ref. [13].) We assume $D=10 \mathrm{kpc}$, and a detector fiducial mass of 1 kton for KamLAND. As written, Eq. (3.1) is for the yield per flavor, assuming that each carries away a portion $E$ of the total binding energy (nominally, $E_{B}=3 \times 10^{53} \mathrm{ergs}$, and $\left.E=E_{B} / 6\right)$. The thermally averaged cross section (the integral of the cross section with normalized Fermi-Dirac distribution) is defined for each $\mathrm{CH}_{2}$ " $\mathrm{mol}$ ecule," and a factor of 2 must be included for electron or free proton targets. The spectrum shape of supernova events which interact in the detector is given by the product of the cross section and a Fermi-Dirac distribution, i.e.,

$$
\frac{d N}{d E_{\nu}} \sim \sigma\left(E_{\nu}\right) \frac{E_{\nu}^{2}}{1+\exp \left(E_{\nu} / T\right)}
$$

For a cross section $\sigma \sim E_{\nu}^{2}$, this peaks at about $4 T$ (for comparison, the average neutrino energy before weighting by the cross section is 3.15T), and the yield $N \sim T$.

Prior to this paper, the largest expected yield in any oil or water detector was from $\bar{\nu}_{e}+p \rightarrow e^{+}+n$. As noted in Sec. II, the total cross sections for charged-current $\bar{\nu}_{e}+p \rightarrow e^{+}+n$ and neutral-current $\nu+p \rightarrow \nu+p$ have similar forms, though the latter is about 4 times smaller. However, this is compensated in the yield by the contributions of all six flavors, as well as the higher temperature assumed for $\nu_{\mu}$ and $\nu_{\tau}(T$ $=8 \mathrm{MeV}$ instead of $5 \mathrm{MeV}$ ). Thus, the total yield from $\nu$ $+p \rightarrow \nu+p$ is larger than that from $\bar{\nu}_{e}+p \rightarrow e^{+}+n$, when the detector threshold is neglected.

Taking into account radiative, recoil, and weak magnetism corrections, the thermally averaged cross section for $\bar{\nu}_{e}+p$ $\rightarrow e^{+}+n$ at $T=5 \mathrm{MeV}$ is $44 \times 10^{-42} \mathrm{~cm}^{2}$ (for 2 protons) [6]. These corrections reduce the thermally averaged cross section by about $20 \%$, and also correct the relation $E_{e}=E_{\nu}$ $-1.3 \mathrm{MeV}$. The total expected yield from this reaction is thus about 310 events in 1 kton.

In Fig. 2, the relative contributions to the spectra of neutrinos that interact in the detector are shown. The integral for the combined yield from $\nu_{\mu}, \nu_{\tau}, \bar{\nu}_{\mu}$, and $\bar{\nu}_{\tau}$ clearly dominates. Further, since the differential cross section favors large $T_{p}$, and since $T_{p} \sim E_{\nu}^{2} / M_{p}$, the corresponding proton recoil 


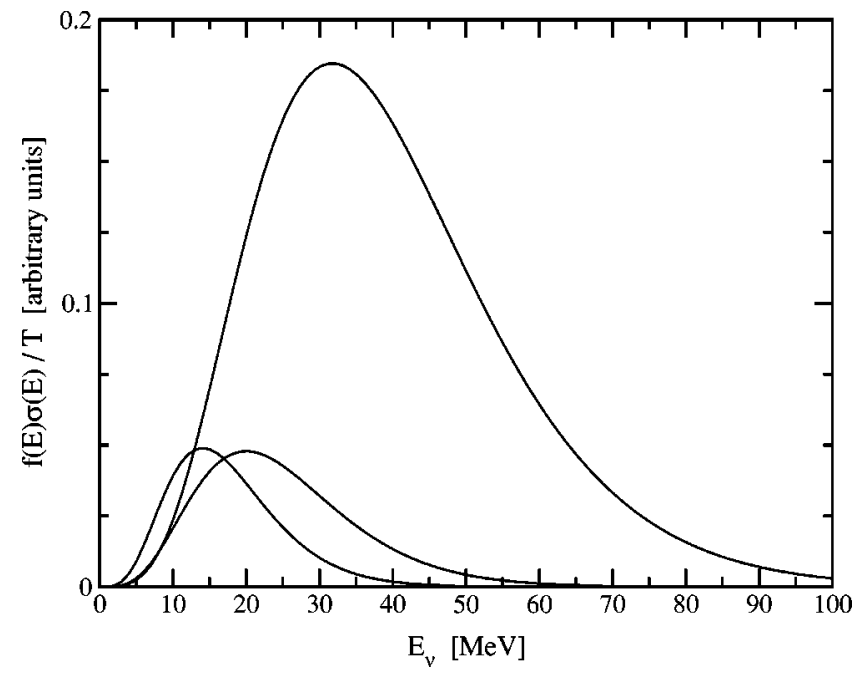

FIG. 2. The relative spectra of neutrinos that interact via neutrino-proton elastic scattering. From left to right in peak position, the curves correspond to $\nu_{e}, \bar{\nu}_{e}$, and the sum of $\nu_{\mu}, \nu_{\tau}, \bar{\nu}_{\mu}$, and $\bar{\nu}_{\tau}$. The flux factors $N_{\nu}=\left(E_{B} / 6\right) /\left\langle E_{\nu}\right\rangle \sim 1 / T$ have been included in the weighting.

kinetic energy spectrum will be much harder, so that they will be even more dominant above a realistic detector threshold.

Since the struck protons in $\nu+p \rightarrow \nu+p$ have a relatively low-energy recoil spectrum, and since realistic detectors have thresholds, it is crucial to consider the proton spectrum in detail, and not just the total yield of neutrinos that interact.

\section{PROTON RECOIL SPECTRUM}

The elastically scattered protons will have kinetic energies of a few MeV. Obviously, these very nonrelativistic protons will be completely invisible in any Cerenkov detector like Super-Kamiokande. However, such small energy depositions can be readily detected in scintillator detectors such as KamLAND and Borexino. We first consider the true proton spectrum, and then in the next section, we consider how this spectrum would appear in a realistic detector.

The true proton spectrum (for one flavor of neutrino) is given by

$$
\frac{d N}{d T_{p}}\left(T_{p}\right)=C \int_{\left(E_{\nu}\right)_{\min }}^{\infty} d E_{\nu} f\left(E_{\nu}\right) \frac{d \sigma}{d T_{p}}\left(E_{\nu}, T_{p}\right),
$$

where $f\left(E_{\nu}\right)$ is a normalized Fermi-Dirac spectrum and the differential cross section is given by Eq. (2.1). For a given $T_{p}$, the minimum required neutrino energy is

$$
\left(E_{\nu}\right)_{\text {min }}=\frac{T_{p}+\sqrt{T_{p}\left(T_{p}+2 M_{p}\right)}}{2} \simeq \sqrt{\frac{M_{p} T_{p}}{2}} .
$$

The normalization constant $C$ is determined by Eq. (3.1), as the integral of Eq. (4.1) over all $T_{p}$ without the $C$ factor is $\langle\sigma\rangle$.

In Fig. 3, we show $d \sigma / d T_{p}$ weighted by normalized Fermi-Dirac distributions of various temperatures, for a

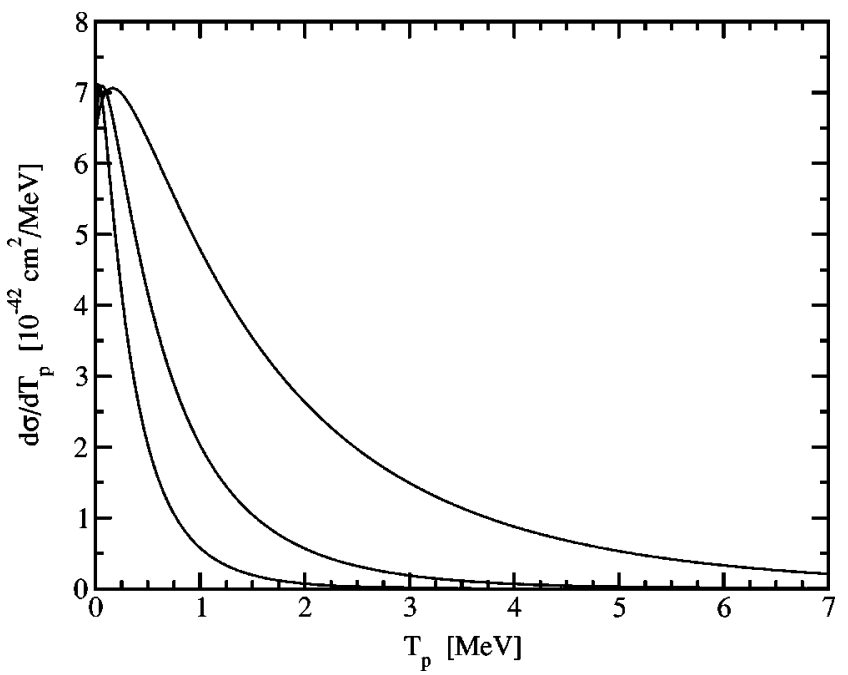

FIG. 3. The thermally averaged differential cross section for Fermi-Dirac distributions of temperature $T=3.5,5,8 \mathrm{MeV}$, from left to right. This illustrates how the proton spectrum changes with the assumed neutrino temperature (since this is a neutral-current cross section, it is flavor-independent).

single neutrino flavor. Throughout this paper, we refer to the $\nu_{e}(T=3.5 \mathrm{MeV}), \bar{\nu}_{e}(T=5 \mathrm{MeV})$, and the combined $\nu_{\mu}$, $\nu_{\tau}, \bar{\nu}_{\mu}$, and $\bar{\nu}_{\tau}(T=8 \mathrm{MeV})$ flavors. Since we know that there are neutrino oscillations, this language is somewhat incorrect. However, our results are totally insensitive to any oscillations among active neutrinos or antineutrinos (since this is a neutral-current cross section), and also to oscillations between active neutrinos and antineutrinos (since the cross section is dominated by the $c_{A}^{2}$ terms). Thus when we refer to the $\nu_{e}$ flavor, we mean "those neutrinos emitted with a temperature $T=3.5 \mathrm{MeV}$, whatever their flavor composition now," etc.

The true proton spectra corresponding to the various flavors are shown in Fig. 4. As seen in the figure, the contributions of $\nu_{e}$ and $\bar{\nu}_{e}$ are quite suppressed relative to the sum of $\nu_{\mu}, \nu_{\tau}, \bar{\nu}_{\mu}$, and $\bar{\nu}_{\tau}$.

\section{QUENCHING}

Low-energy protons lose energy very quickly by ionization. The energy loss rate $d E / d x$ of nonrelativistic particles scales roughly as $d E / d x \sim-z^{2} / \beta^{2}$ in this energy range [14], where $z$ is the particle charge and $\beta$ its velocity. In contrast with the usual $-2 \mathrm{MeV} / \mathrm{g} / \mathrm{cm}^{2}$ for a minimum-ionizing particle, for few-MeV protons, $d E / d x \sim-100 \mathrm{MeV} / \mathrm{g} / \mathrm{cm}^{2}$. Thus even a $10 \mathrm{MeV}$ proton will be brought to rest in less than about $0.1 \mathrm{~cm}$. In contrast, the hadronic interaction length for the proton to scatter from a free or bound nucleon is of order $1 \mathrm{~cm}$ or larger. Thus the hadronic energy losses can be totally neglected; see also Fig. 23.1 of Ref. [14]. Because of the nonlinear response of the detector to proton recoil energies, as we are about to describe, it is important that the original proton energy is not shared among two or more protons, i.e., from elastic hadronic scattering.

In a scintillator, there is generally an efficient transfer 


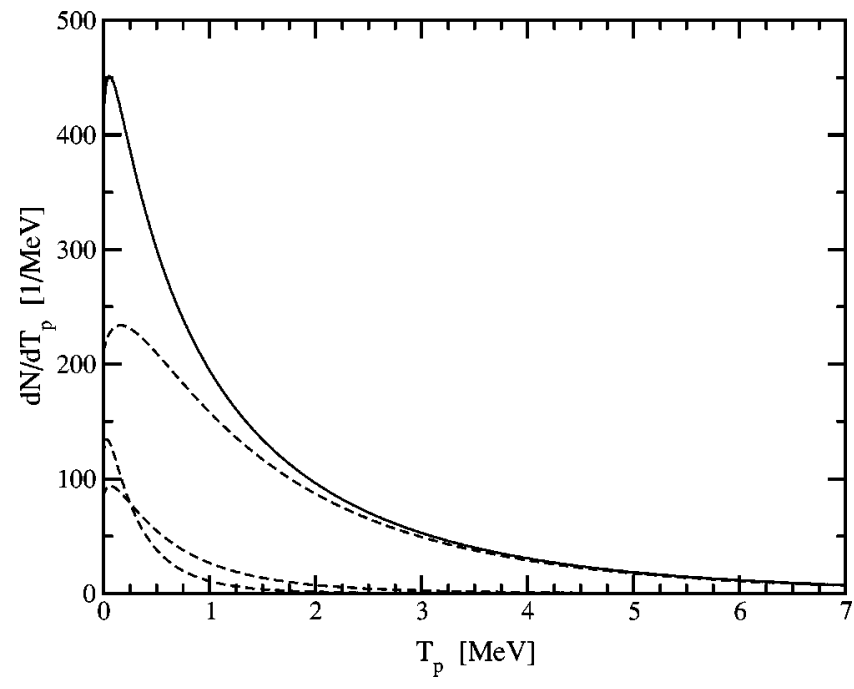

FIG. 4. The true proton spectrum in KamLAND, for a standard supernova at $10 \mathrm{kpc}$. In order of increasing maximum kinetic energy, the contributions from $\nu_{e}, \bar{\nu}_{e}$, and the sum of $\nu_{\mu}, \nu_{\tau}, \bar{\nu}_{\mu}$, and $\bar{\nu}_{\tau}$ are shown with dashed lines. The solid line is the sum spectrum for all flavors. Taking the detector properties into account substantially modifies these results, as shown below.

between the ionization loss of a charged particle and the detectable scintillation light observed by phototubes. For example, in KamLAND, there are approximately 200 detected photoelectrons per $\mathrm{MeV}$ deposited for a minimum-ionizing particle like an electron [15].

However, for highly ionizing particles like low-energy protons, the light output is reduced or "quenched" relative to the light output for an electron depositing the same amount of energy. The observable light output $E_{\text {equiv }}$ (i.e., equivalent to an electron of energy $E_{\text {equiv }}$ ) is given by Birk's law [16]:

$$
\frac{d E_{\text {equiv }}}{d x}=\frac{d E / d x}{1+k_{B}(d E / d x)}
$$

where $k_{B}$ is a constant of the scintillation material, and $d E / d x$ is the energy deposition rate, now in $\mathrm{MeV} / \mathrm{cm}$ (and defined to be positive). We assume $k_{B} \simeq 0.015 \mathrm{~cm} / \mathrm{MeV}$ for KamLAND [15]. For small $d E / d x$, the measured light output of a proton is equivalent to that from an electron of the same energy. But for $d E / d x \sim 100 \mathrm{MeV} / \mathrm{cm}$, the two terms in the denominator are comparable, and the light output is reduced. At still higher $d E / d x$, then $d E_{\text {equiv }} / d x$ tends to a constant. Birk's law can thus reflect a saturation effect: once $d E / d x$ is large, making it larger does not increase the light output. Effectively, if all scintillation molecules along the path of the particle are already excited, any further energy deposition is not converted to visible scintillation light.

The proton quenching factor was calculated by integrating Eq. (5.1) with tables [17] of $d E / d x$ for protons in the KamLAND oil-scintillator mixture [15]:

$$
E_{\text {equiv }}\left(T_{p}\right)=\int_{0}^{T p} \frac{d E}{1+k_{B}(d E / d x)} .
$$

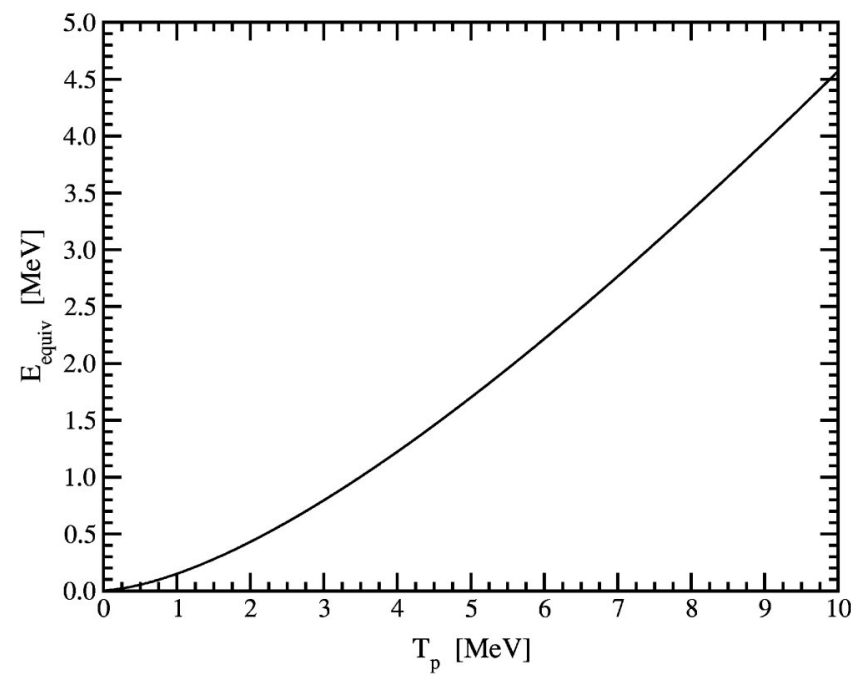

FIG. 5. The quenched energy deposit (equivalent electron energy) as a function of the proton kinetic energy. The KamLAND detector properties are assumed.

The observed energy in terms of the proton kinetic energy is shown in Fig. 5. Thus the proton quenching factor $\left(E_{\text {equiv }} / T_{p}\right.$ ) is roughly $1 / 2$ at $10 \mathrm{MeV}, 1 / 3$ at $6 \mathrm{MeV}, 1 / 4$ at 3 $\mathrm{MeV}$, and so on. The detector response is nonlinear, though in a well-understood way. A similar calculation using $\alpha$ particles recovered the quenching factor of approximately 1/14 noted in Ref. [15]. Since the energy deposition scales roughly as $d E / d x \sim z^{2} / \beta^{2}$, quenching for alpha particles is much worse than for protons of the same kinetic energy, since $d E / d x$ is approximately $4 \times 4=16$ times larger. Our results for the proton quenching factor are in good agreement with direct measurements in a variety of scintillators $[16,18]$.

Using the quenching function shown in Fig. 5, we can transform the true proton spectrum shown in Fig. 4 into the expected measured proton spectrum, shown in Fig. 6. If the quenching factor were a constant, it would simply change the units of the $T_{p}$ axis. However, it is nonlinear, and reduces the light output of the lowest recoils the most. This increases the effect, shown in previous figures, that the measurable contribution from $\nu_{e}$ and $\bar{\nu}_{e}$ is highly suppressed relative to the sum of $\nu_{\mu}, \nu_{\tau}, \bar{\nu}_{\mu}$, and $\bar{\nu}_{\tau}$.

As shown, quenching distorts the spectra according to a known nonlinear function. It also reduces the number of events above threshold. The anticipated threshold in KamLAND is $0.2 \mathrm{MeV}$ electron equivalent energy (strictly speaking, KamLAND and Borexino have somewhat higher target thresholds of approximately 0.28 and $0.25 \mathrm{MeV}$, set by background rates; over the short duration of the supernova pulse, much higher background rates can be tolerated). With the expected proton quenching, this corresponds to a threshold on the true proton kinetic energy of $1.2 \mathrm{MeV}$. The number of events above this threshold for each flavor appears in Table I. Above an electron equivalent threshold of $0.2 \mathrm{MeV}$, the neutrino-proton elastic scattering yields from $\nu_{e}$ and $\bar{\nu}_{e}$ are quite small. Thus the measured proton spectrum will primarily reflect the shape of the underlying Fermi-Dirac spectrum for the sum of $\nu_{\mu}, \nu_{\tau}, \bar{\nu}_{\mu}$, and $\bar{\nu}_{\tau}$. Of course, this has been 


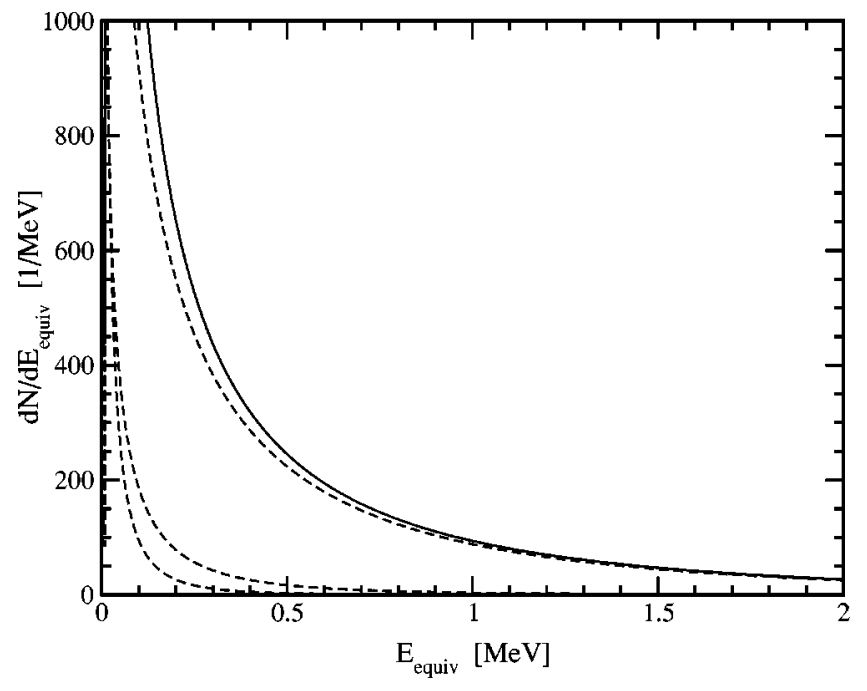

FIG. 6. Analogous to Fig. 4; the struck proton spectrum for the different flavors, but with quenching effects taken into account. In order of increasing maximum kinetic energy, the contributions from $\nu_{e}, \bar{\nu}_{e}$, and the sum of $\nu_{\mu}, \nu_{\tau}, \bar{\nu}_{\mu}$, and $\bar{\nu}_{\tau}$ are shown with dashed lines. The solid line is the sum spectrum for all flavors. We assume a 1 kton detector mass for KamLAND.

convolved with both the differential cross section (which gives a range of $T_{p}$ for a given $E_{v}$ ), and also the effects of quenching. However, as we will show, the properties of the initial neutrino spectrum can still be reliably deduced. The numbers of events above a given electron equivalent threshold are shown in Fig. 7.

\section{BACKGROUNDS}

In this section, we consider several backgrounds to the signal of neutrino-proton elastic scattering from supernova $\nu_{\mu}, \nu_{\tau}, \bar{\nu}_{\mu}$, and $\bar{\nu}_{\tau}$.

The first is neutrino-proton elastic scattering from $\nu_{e}$ and $\bar{\nu}_{e}$. As shown above, in particular in Figs. 6 and 7, this contribution is minimal above the expected threshold. We

TABLE I. Numbers of events in KamLAND (1 kton mass assumed) above the noted thresholds for a standard supernova at 10 $\mathrm{kpc}$, for the separate flavors or their equivalents after oscillations. Oscillations do not change the number of neutrinos at a given energy, and the neutral-current yields are insensitive to the neutrino flavor. Equipartition among the six flavors is assumed (see the text for discussion). The thresholds are in electron equivalent energy, and correspond to minimum true proton kinetic energies of 0 and 1.2 MeV. As discussed in Sec. II, weak magnetism corrections are not included.

\begin{tabular}{lcc}
\hline \hline Neutrino Spectrum & $E_{t h r}=0$ & $0.2 \mathrm{MeV}$ \\
\hline$\nu: T=3.5 \mathrm{MeV}$ & 57 & 3 \\
$\bar{\nu}: T=5 \mathrm{MeV}$ & 80 & 17 \\
$2 \nu: T=8 \mathrm{MeV}$ & 244 & 127 \\
$2 \bar{\nu}: T=8 \mathrm{MeV}$ & 243 & 126 \\
All & 624 & 273 \\
\hline \hline
\end{tabular}

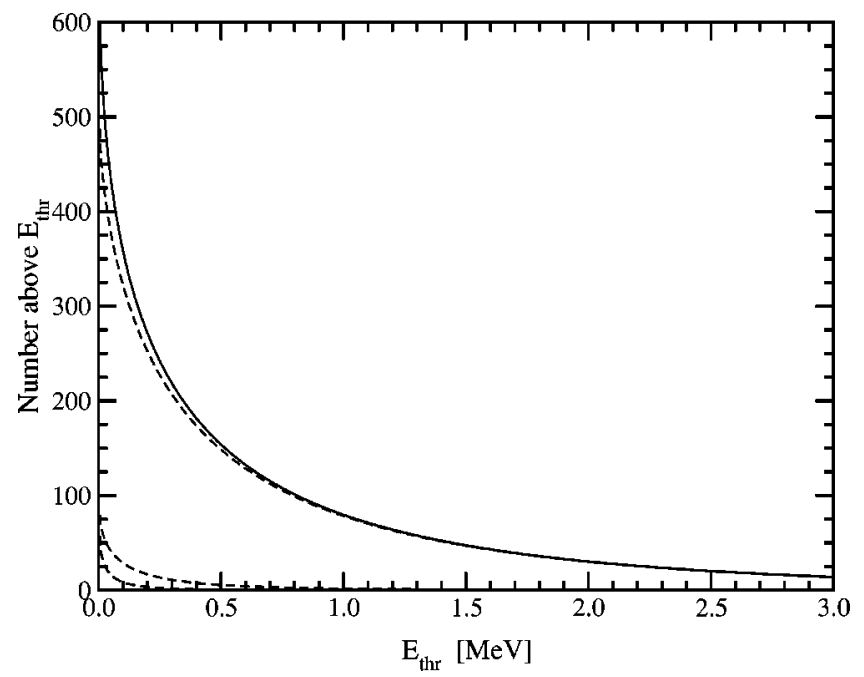

FIG. 7. The number of events above threshold in KamLAND as a function of $E_{t h r}$ in electron equivalent energy $E_{\text {equiv }}$. In order of increasing maximum kinetic energy, the contributions from $\nu_{e}, \bar{\nu}_{e}$, and the sum of $\nu_{\mu}, \nu_{\tau}, \bar{\nu}_{\mu}$, and $\bar{\nu}_{\tau}$ are shown with dashed lines. The solid line is for the sum of all flavors. We assume a threshold of $0.2 \mathrm{MeV}$ electron equivalent energy in KamLAND.

assume that it can be statistically subtracted using knowledge of $\nu_{e}$ and $\bar{\nu}_{e}$ temperatures measured in charged-current reactions and do not consider it further.

The second comes from a variety of other charged-current supernova neutrino signals in the detector. As noted above, approximately 310 events are expected from $\bar{\nu}_{e}+p \rightarrow e^{+}$ $+n$ [6]. These events can easily be identified by the tight coincidence (roughly a few times $10 \mathrm{~cm}$ in position, and 0.2 $\mathrm{ms}$ in time) in the detection of the high-energy (about 20 $\mathrm{MeV}$ ) positrons and the subsequent neutron captures on protons (2.2 MeV gamma). There are also charged-current reactions of $\nu_{e}$ and $\bar{\nu}_{e}$ on ${ }^{12} \mathrm{C}$, proceeding almost exclusively to the ground states of ${ }^{12} \mathrm{~N}$ and ${ }^{12} \mathrm{~B}$, respectively. About 10 events are expected, and possibly a few times more if oscillations effectively swap spectra [15]. However, these events can be identified by the subsequent ${ }^{12} \mathrm{~N}$ and ${ }^{12} \mathrm{~B}$ beta decays, with lifetimes of order $10 \mathrm{~ms}$ and electron endpoints of order $15 \mathrm{MeV}$. The total yield from neutrino-electron elastic scattering (technically, mixed charged- and neutral-current) is expected to be about 20 events. We assume that these events can be statistically subtracted from the spectrum, or that particle identification by pulse-shape-discrimination (PSD) will be possible.

The third comes from other neutral-current supernova neutrino signals in the detector. The best-known is the superallowed neutral-current excitation of the $15.11 \mathrm{MeV}$ state in ${ }^{12} \mathrm{C}$, which decays by gamma emission. About 60 events are expected, and they will be easily identified by their narrow spectrum at $15.11 \mathrm{MeV}$ [15]. There are also inelastic neutral-current excitations of ${ }^{12} \mathrm{C}$ that decay by particle emission. The yield from all channels that emit a proton is about 45 events, using the cross sections and branching ratios of Ref. [19]. Some fractions, probably most, will be above threshold and will add to the signal of low-energy 
protons. Though the proton spectra for these reactions have not been published, we assume that their contribution will be known and can be subtracted. The yield from all channels that emit a neutron is about 20 events, again using the results of Ref. [19]. These neutrons will be captured, giving 2.2 $\mathrm{MeV}$ gamma rays. These inelastic neutral-current reactions with proton and neutron emission have not previously been recognized as supernova neutrino detection channels. Finally, since a $50 \mathrm{MeV}$ neutrino corresponds to a wavelength of 4 $\mathrm{fm}$, about the diameter of a carbon nucleus, there can also be coherent neutral-current scattering of the whole nucleus [20]. The number of events is very large, coincidentally as large as the total neutrino-proton elastic scattering yield (neglecting the detector threshold). However, the expected recoil kinetic energies are of course about 12 times smaller than for free protons. Additionally, since it is spin-independent vector scattering, the smallest recoil energies are favored. (In contrast, neutrino-proton elastic scattering is spin-dependent, and the proton spin is flipped in the scattering.) The recoil carbon ions will be very heavily quenched, and so this signal is unobservable in a detector like KamLAND.

The fourth comes from cosmic-ray induced detector backgrounds. Because it is located deep underground, the muon rate in the KamLAND scintillator is only about $0.3 \mathrm{~Hz}$, and so all muon-related backgrounds are very small over the short duration of the supernova burst [15].

The fifth and most serious comes from low-energy radioactivities in and around the detector. Normally, these are not a concern for relatively high-energy supernova events. However, here we are considering signals down to about $0.2 \mathrm{MeV}$ detected energy, where many different radioactive backgrounds contribute. At present, KamLAND is configured to detect few-MeV reactor antineutrinos via the coincidence between the prompt positrons and the delayed neutron captures, and low singles backgrounds above $0.2 \mathrm{MeV}$ are not required. Published data on the KamLAND background spectrum are not yet available. However, if KamLAND is to be eventually used for detecting ${ }^{7} \mathrm{Be}$ solar neutrinos by neutrino-electron scattering, then the background in this energy range will have to be reduced to about $10^{-3} \mathrm{~Hz}$, the rate of solar neutrino events expected (similar considerations hold for Borexino). For the supernova signal discussed in this paper, a much larger background rate of about $1 \mathrm{~Hz}$ could be tolerated. This rate is set by the consideration of being much less than $(300$ events $/ 10 \mathrm{~s})=30 \mathrm{~Hz}$.

Therefore, in what follows we consider just the main signal, and neglect backgrounds.

\section{PROTON SPECTRUM FITS}

In this section, we show how the measured proton spectrum can be used to separately determine the total energy of the $\nu_{\mu}, \nu_{\tau}, \bar{\nu}_{\mu}$, and $\bar{\nu}_{\tau}$ neutrinos and their time-averaged temperature. The total number of detected events is proportional to the portion of the total binding energy carried away by these four flavors, and we denote this by $E^{\text {tot }}$ (note that this is not the total binding energy $\left.E_{B}\right)$. For a standard supernova, $E^{t o t}=4\left(E_{B} / 6\right)=2 / 3 E_{B} \simeq 2 \times 10^{53}$ ergs. We denote the temperature of these four flavors by $T$. If only the total yield were measured, as for most neutral-current reactions, there would be an unresolved degeneracy between $E^{\text {tot }}$ and $T$, since

$$
N \sim E^{t o t} \frac{\langle\sigma\rangle}{T}
$$

Note that for $\sigma \sim E_{\nu}^{n}$, then $\langle\sigma\rangle \sim T^{n}$. For $\nu+d \rightarrow \nu+p+n$ in SNO, for example, $\sigma \sim E^{2}$, so $N \sim E^{t o t} T$. Thus for a given measured number of events, one would only be able to define a hyperbola in the plane of $E^{t o t}$ and $T$. The scaling is less simple here because of threshold effects, but the idea is the same.

Here we have crucial information on the shape of the neutrino spectrum, revealed through the proton spectrum. To remind the reader, in most neutral-current reactions there is no information on the neutrino energy, e.g., one only counts the numbers of thermalized neutron captures, or measures nuclear gamma rays (the energies of which depend only on nuclear level splittings).

In this section, we perform quantitative tests of how well the parameters $E^{t o t}$ and $T$ can be determined from the measured proton spectrum. (We did also investigate the effects of a chemical potential in the Fermi-Dirac distribution, but found that it had little effect. This is simply because the cross section is not rising quickly enough to see the tail of the thermal distribution in detail [21].) Of course, if the distance to the supernova is not known, then we are effectively fitting for $E^{t o t} / D^{2}$.

We performed Monte Carlo simulations of the supernova signal in KamLAND and made chi-squared fits to determine $E^{t o t}$ and $T$ for each fake supernova. To perform the fits, we started with an "ideal" spectrum, as described by the integral:

$$
\begin{aligned}
\left(\frac{d N}{d T_{p}}\right)_{\text {ideal }}= & C \int_{0}^{\infty} d T_{p}^{\prime} G\left(T_{p}^{\prime} ; T_{p}, \delta T_{p}\right) \\
& \times \int_{\left(E_{\nu}\right)_{\text {min }}}^{\infty} d E_{\nu} f\left(E_{\nu}\right) \frac{d \sigma}{d T_{p}^{\prime}}\left(E_{\nu}, T_{p}^{\prime}\right),
\end{aligned}
$$

where the inner integral is as in Eq. (4.1), with the addition that quenching corrections are applied to $T_{p}^{\prime}$ after convolution with $f\left(E_{\nu}\right)$. For the Gaussian energy resolution $G\left(T_{p}^{\prime} ; T_{p}, \delta T_{p}\right)$, we used $\delta T_{p}=0.1 \sqrt{T_{p} /(1 \mathrm{MeV})}$ [15]. Because the proton spectrum has already been smeared by the neutrino spectrum and the differential cross section, the Gaussian energy resolution has only a minor effect. The normalization constant $C$ is given by comparison to Eq. (3.1). Example spectra are shown in Fig. 8.

Using $\left(d N / d T_{p}\right)_{\text {ideal }}$, we binned the spectrum by the following integral:

$$
N_{i}=\int_{\left(E_{\min }\right)_{i}}^{\left(E_{\max }\right)_{i}} d T_{p}\left(\frac{d N}{d T_{p}}\right)_{\text {ideal }}
$$

where $N_{i}$ is the number of events in bin $i$, and $\left(E_{\text {min }}\right)_{i}$ and $\left(E_{\max }\right)_{i}$ are the minimum and maximum energies for bin $i$. 


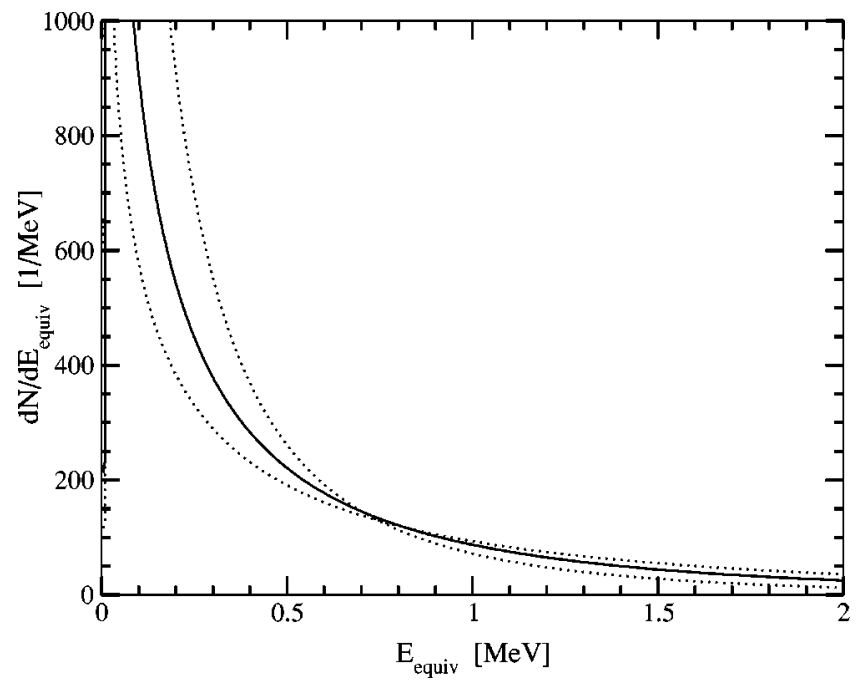

FIG. 8. Example spectra with different values of $E^{\text {tot }}$ and $T$, all chosen to give the same number of events above an electron equivalent threshold of $0.2 \mathrm{MeV}$ (true proton energy $1.2 \mathrm{MeV}$ ) in KamLAND. Though not shown in this figure, the spectrum above 2 $\mathrm{MeV}$ is included in our analysis. At the $0.2 \mathrm{MeV}$ point, from left to right these correspond to $\left(E^{t o t}, T\right)=(4.2,6),(2.0,8),(1.4,10)$, respectively, with $E^{\text {tot }}$ in $10^{53} \mathrm{ergs}$ and $T$ in $\mathrm{MeV}$. (Thus with the standard $E^{\text {tot }}=2 \times 10^{53} \mathrm{ergs}$, the number of events above threshold with $T=6 \mathrm{MeV}$ is $2.0 / 4.2$ times the number with $T=8 \mathrm{MeV}$; with $T=10 \mathrm{MeV}$, it is 2.0/1.4 times the number with $T=8 \mathrm{MeV}$.)

Eight bins of variable width were used, chosen to contain roughly the same number of expected events per bin. For a chosen $E^{\text {tot }}$ and $T$, this was the starting point of our Monte Carlo calculation (and the bin boundaries were kept fixed). For each fake supernova, we sampled the number of events in each of these bins according to the appropriate Poisson distributions. The resulting spectrum was as one might obtain from a single supernova, given the finite number of events expected. We then varied $E^{t o t}$ and $T$ in Eq. (7.3) until the values that best fit the fake spectral data were determined. For a given set of assumed $E^{t o t}$ and $T$, this procedure was repeated many times. The distributions of the final $E^{\text {tot }}$ and $T$ thus reveal the expected errors on fitting $E^{t o t}$ and $T$ for a single real future supernova.

Three examples are shown in Fig. 9, where one can see that $E^{t o t}$ and $T$ can each be determined with roughly $10 \%$ error. These errors scale as $1 / \sqrt{N}$, where $N$ is the total number of events (i.e., if one imagines a detector of a different mass or a different assumed supernova distance). If the distance were completely uncertain, one would not be able to determine $E^{t o t}$. However, after marginalizing over the unknown $E^{\text {tot }}$ (i.e., projecting these scatterplots onto the $T$ axis), one would still obtain a good measurement of $T$.

\section{DISCUSSION AND CONCLUSIONS}

We have shown that neutrino-proton elastic scattering, previously unrecognized as a useful detection reaction for low-energy neutrinos, in fact has a yield for a supernova comparable to $\bar{\nu}_{e}+p \rightarrow e^{+}+n$, even after taking into account

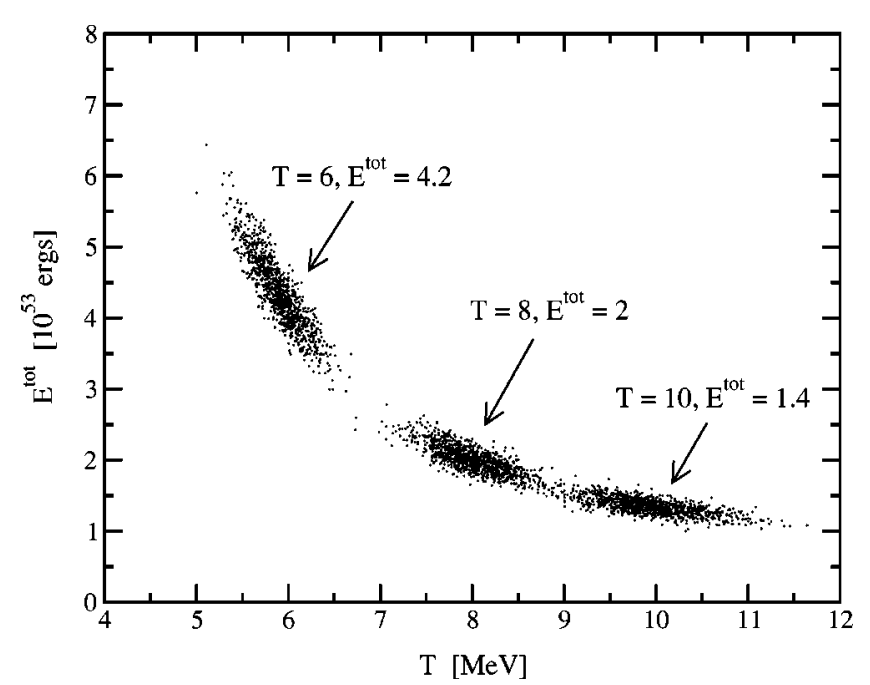

FIG. 9. Scatterplot of $10^{3}$ fitted values, in the $E^{\text {tot }}$ and $T$ plane, for the labeled "true" values, where $E^{\text {tot }}$ is the total portion of the binding energy carried away by the sum of $\nu_{\mu}, \nu_{\tau}, \bar{\nu}_{\mu}$, and $\bar{\nu}_{\tau}$, and $T$ is their temperature. The values of $E^{\text {tot }}$ and $T$ were chosen such that the numbers of events above threshold were the same. The measured shape of the proton spectrum breaks the degeneracy between these two parameters. Without that spectral information, one could not distinguish between combinations of $E^{\text {tot }}$ and $T$ along the band in this plane that our three example regions lie along.

the quenching of the proton scintillation light and assuming a realistic detector threshold.

In addition, the measured proton spectrum is related to the incident neutrino spectrum. We have shown explicitly that one can separately measure the total energy and temperature of $\nu_{\mu}, \nu_{\tau}, \bar{\nu}_{\mu}$, and $\bar{\nu}_{\tau}$, each with uncertainty of order $10 \%$ in KamLAND. This greatly enhances the importance of detectors like KamLAND and Borexino for detecting supernova neutrinos.

For Borexino, the useful volume for supernova neutrinos is $0.3 \mathrm{kton}$, and the hydrogen to carbon ratio in the pure pseudocumene $\left(\mathrm{C}_{9} \mathrm{H}_{12}\right)$ is 1.3:1 [22], so there are about 4.7 times fewer free proton targets than assumed for KamLAND. However, the quenching is less in pure scintillator (KamLAND is about $20 \%$ pseudocumene and $80 \%$ paraffin oil [15]), and the errors on $E^{\text {tot }}$ and $T$ scale as $1 / \sqrt{N}$, so that the precision in Borexino should be about $20 \%$ or better.

Other techniques for bolometric measurements of supernova neutrino fluxes have been studied. Detectors for elastic neutral-current neutrino scattering on electrons [23] and coherently on whole nuclei [20] have been discussed, but never built. If neutrino oscillations are effective in swapping spectra, then the temperature of the "hot" flavors may be revealed in the measured positron spectrum from $\bar{\nu}_{e}+p \rightarrow e^{+}$ $+n$; two recent studies have shown very good precision ( $\$ 5 \%$ ) for measuring the temperatures and the total binding energy $[24,25]$. However, they assumed exact energy equipartition among the six neutrino flavors, whereas the uncertainty on equipartition is at least 50\% [11]. Nevertheless, under less restrictive assumptions, this technique may play a complementary role. Finally, since for different cross sections, the neutral-current yields depend differently on tem- 
perature, comparison of the yields may provide some information [26]. However, there are caveats. In neutrino-electron scattering, the neutrino energy is not measured because the neutrino-electron angle is much less than the angular resolution due to multiple scattering. The scattered electrons, even those in a forward cone, sit on a much larger background of $\bar{\nu}_{e}+p \rightarrow e^{+}+n$ events, so it is difficult to measure their spectrum [27]; also, their total yield is only weakly dependent on temperature. At the other extreme (see Fig. 3 of Ref. [26]), the yield of neutral-current events [28] on ${ }^{16} \mathrm{O}$ depends strongly on a possible chemical potential term in the thermal distribution.

It is important to note that the detection of recoil protons from neutron-proton elastic scattering at several $\mathrm{MeV}$ has been routinely accomplished in scintillator detectors (see, e.g., Ref. [18]). Since both particles are massive, the proton will typically take half of the neutron energy. This reaction provides protons in the same energy range as those struck in neutrino-proton elastic scattering with $E_{\nu} \sim 30 \mathrm{MeV}$. This is a very important proof of concept for all aspects of the detection of low-energy protons.

Though low-energy backgrounds will be challenging, it is also important to note that the background requirements for detecting the supernova signal are approximately 3 orders of magnitude less stringent than those required for detecting solar neutrinos in the same energy range (taking quenching into account for our signal). Borexino has been designed to detect very low-energy solar neutrinos, and KamLAND hopes to do so in a later phase of the experiment.

These measurements would be considered in combination with similar measurements for $\nu_{e}$ and $\bar{\nu}_{e}$ from chargedcurrent reactions in other detectors. Separate measurements of the total energy and temperature for each flavor will be invaluable for comparing to numerical supernova models. They will also be required to make model-independent studies of the effects of neutrino oscillations. If the total energy release $E_{B}$ in all flavors has been measured, then

$$
E_{B} \simeq \frac{3}{5} \frac{G M_{N S}^{2}}{R_{N S}}
$$

thus allowing a direct and unique measurement of the newly formed neutron star properties, principally the mass $M_{N S}$ [29].

\section{ACKNOWLEDGMENTS}

We thank Felix Boehm, Laura Cadonati, Frank Calaprice, Mark Chen, Chuck Horowitz, Thomas Janka, Glenn HortonSmith, Loren Hoffman, Bob McKeown, Marianne Neff, Lothar Oberauer, Stephen Parke, Andreas Piepke, Georg Raffelt, Junpei Shirai, Fumihiko Suekane, Rex Tayloe, Bryan Tipton, and Bruce Vogelaar for discussions. J.F.B. was supported by Caltech and Fermilab. Fermilab is operated by URA under DOE contract No. DE-AC02-76CH03000. J.F.B. was additionally supported by NASA under NAG5-10842. W.M.F. was supported by the SURF Program at Caltech. This work was supported in part by the U.S. Department of Energy under Grant No. DE-FG03-88ER40397 at Caltech.
[1] J.F. Beacom, http://mocha.phys.washington.edu/ 〜int_talk/WorkShops/Neutrino2000/People/Beacom_J/; W.M. Farr, http://www.aps.org/meet/DNP00/baps/abs/S750024.html; P. Vogel, Prog. Part. Nucl. Phys. 48, 29 (2002).

[2] S. Weinberg, Phys. Rev. D 5, 1412 (1972).

[3] L.A. Ahrens et al., Phys. Rev. D 35, 785 (1987).

[4] C.H. Llewellyn Smith, Phys. Rep. 3, 261 (1972); S.M. Bilenky and J. Hosek, ibid. 90, 73 (1982).

[5] C.J. Horowitz, Phys. Rev. D 65, 043001 (2002).

[6] P. Vogel and J.F. Beacom, Phys. Rev. D 60, 053003 (1999).

[7] D.H. Beck and R.D. McKeown, Annu. Rev. Nucl. Part. Sci. 51, 189 (2001); R.D. McKeown and M.J. Ramsey-Musolf, hep-ph/0203011.

[8] L. Wolfenstein, Phys. Rev. D 19, 3450 (1979); G.T. Garvey, W.C. Louis, and D.H. White, Phys. Rev. C 48, 761 (1993); C.J. Horowitz, H.C. Kim, D.P. Murdock, and S. Pollock, ibid. 48, 3078 (1993); M.B. Barbaro, A. De Pace, T.W. Donnelly, A. Molinari, and M.J. Musolf, ibid. 54, 1954 (1996); M.J. Savage and J. Walden, Phys. Rev. D 55, 5376 (1997); W.M. Alberico, S.M. Bilenky, and C. Maieron, Phys. Rep. 358, 227 (2002).

[9] R. Tayloe, Nucl. Phys. B (Proc. Suppl.) 105, 62 (2002).

[10] J.R. Wilson and R.W. Mayle, Phys. Rep. 227, 97 (1993); M. Herant, W. Benz, W.R. Hix, C.L. Fryer, and S.A. Colgate, Astrophys. J. 435, 339 (1994); A. Burrows, J. Hayes, and B.A. Fryxell, ibid. 450, 830 (1995); M. Rampp and H.T. Janka,
Astrophys. J. Lett. 539, L33 (2000); A. Mezzacappa, M. Liebendorfer, O.E. Messer, W.R. Hix, F.K. Thielemann, and S.W. Bruenn, Phys. Rev. Lett. 86, 1935 (2001); C.L. Fryer and A. Heger, Astrophys. J. 541, 1033 (2000).

[11] G.G. Raffelt, Nucl. Phys. B (Proc. Suppl.) 110, 254 (2002).

[12] J.F. Beacom, R.N. Boyd, and A. Mezzacappa, Phys. Rev. D 63, 073011 (2001).

[13] M.K. Sharp, J.F. Beacom, and J.A. Formaggio, Phys. Rev. D (to be published), hep-ph/0205035.

[14] Particle Data Group, D.E. Groom et al., Eur. Phys. J. C 15, 1 (2000).

[15] A. Suzuki, in Lepton and Baryon Number Violation Particle Physics, Astrophysics, and Cosmology, edited by H.V. Klapdor-Kleingrothaus and I.V. Krivosheina (Institute of Physics, Philadelphia, 1999); S.J. Freedman et al., Proposal for US Participation in KamLAND, 1999; A. Piepke, Nucl. Phys. B (Proc. Suppl.) 91, 99 (2001); http://www.awa.tohoku.ac.jp/html/KamLAND/

[16] W.R. Leo, Techniques for Nuclear and Particle Physics Experiments, 2nd ed. (Springer-Verlag, Berlin, 1994).

[17] NIST PSTAR Database: http://physics.nist.gov/PhysRefData/ Star/Text/

[18] J.B. Czirr, D.R. Nygren, and C.D. Zafiratos, Nucl. Instrum. Methods 31, 226 (1964); K.H. Maier and J. Nitschke, ibid. 59, 227 (1968); R.L. Craun and D.L. Smith, ibid. 80, 239 (1970); 
D.J. Ficenec, S.P. Ahlen, A.A. Marin, J.A. Musser, and G. Tarle, Phys. Rev. D 36, 311 (1987); S. Mouatassim, G.J. Costa, G. Guillaume, B. Heusch, A. Huck, and M. Moszynski, Nucl. Instrum. Methods Phys. Res. A 359, 530 (1995); B. Achkar et al., Phys. Lett. B 374, 243 (1996).

[19] S.E. Woosley, D.H. Hartmann, R.D. Hoffman, and W.C. Haxton, Astrophys. J. 356, 272 (1990); E. Kolbe, K. Langanke, S. Krewald, and F.K. Thielemann, Nucl. Phys. A540, 599 (1992); E. Kolbe, K. Langanke, S. Krewald, and F.K. Thielemann, Phys. Rep. 227, 37 (1993).

[20] D.Z. Freedman, Phys. Rev. D 9, 1389 (1974); A. Drukier and L. Stodolsky, ibid. 30, 2295 (1984).

[21] J.F. Beacom and P. Vogel, Phys. Rev. D 58, 093012 (1998).

[22] L. Cadonati, F.P. Calaprice, and M.C. Chen, Astropart. Phys. 16, 361 (2002).
[23] B. Cabrera, L.M. Krauss, and F. Wilczek, Phys. Rev. Lett. 55, 25 (1985).

[24] V. Barger, D. Marfatia, and B.P. Wood, hep-ph/0112125.

[25] H. Minakata, H. Nunokawa, R. Tomas, and J.W. Valle, hep-ph/0112160.

[26] J.F. Beacom, in Neutrinos in the New Millennium, edited by G. Domokos and S. Kovesi-Domokos (World Scientific, Singapore, 2000), hep-ph/9909231. Note that the version in the proceedings volume contains no minus signs; the version on the arXiv is correct.

[27] J.F. Beacom and P. Vogel, Phys. Rev. D 60, 033007 (1999).

[28] K. Langanke, P. Vogel, and E. Kolbe, Phys. Rev. Lett. 76, 2629 (1996); J.F. Beacom and P. Vogel, Phys. Rev. D 58, 053010 (1998).

[29] J.M. Lattimer and M. Prakash, Astrophys. J. 550, 426 (2001). 